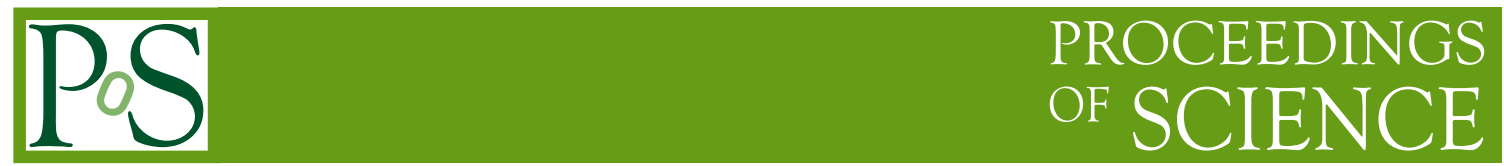

\title{
Recent progress on high order calculations and matching to parton showers
}

\author{
Emanuele $\mathbf{R e}^{a, 1, *}$ \\ ${ }^{a}$ LAPTh, Université Grenoble Alpes, Université Savoie Mont Blanc, CNRS, \\ F-74940 Annecy, France \\ E-mail: emanuele.re@lapth.cnrs.fr \\ I give an overview of the recent progress on the matching of fixed-order calculations and parton \\ showers. The focus is on the matching with NNLO QCD corrections as well as with NLO EW \\ ones.
}

The Ninth Annual Conference on Large Hadron Collider Physics - LHCP2021

7-12 June 2021

Online

${ }^{1}$ Preprint number: LAPTH-Conf-029/21

* Speaker 


\section{Introduction}

The current experimental precision reached by the LHC experimental collaborations (and even more the future prospects) requires theoretical predictions whose formal accuracy goes beyond the computation of NLO QCD corrections and their matching to parton showers ( $\mathrm{NLO}_{\mathrm{QCD}}+\mathrm{PS}$ ). Including (N)NNLO QCD corrections, and NLO EW ones (or combination thereof), is crucial, as often it is only through such predictions that a comparison between data and theory is made possible without being limited by the quality of theoretical predictions. In the rest of this review, I'll summarize the recent progress in the matching of NNLO QCD computations with parton showers $\left(\mathrm{NNLO}_{\mathrm{QCD}}+\mathrm{PS}\right)$, and in the inclusion of $\mathrm{EW}$ corrections in event generators.

\section{Matching NNLO QCD corrections with Parton Showers}

The issue of matching NNLO QCD corrections to parton showers has been already addressed by different groups, and $\mathrm{NNLO}_{\mathrm{QCD}}+\mathrm{PS}$ results have been obtained with four methods: "reweighted MiNLO'" [1, 2], Geneva [3, 4], Unnlops [5], MiNNLOPS [6, 7]. ${ }^{1}$ Very schematically, the core ideas of these four methods can be summarized as follows:

- "reweighted" MiNLO' and MiNNLO ${ }_{\mathrm{PS}}$ are based on the merging of $\mathrm{NLO}_{\mathrm{QCD}}+\mathrm{PS}$ results for $p p \rightarrow F$ and $p p \rightarrow F+j$ production, where $F$ denotes a generic color-singlet final state. Such merging is obtained without any external resolution parameter, through the use of information known from transverse-momentum resummation.

- In Geneva, one constructs IR-finite events using a resolution parameter (until recently, the " $N$-jettiness" $\tau_{N}$ ) whose resummation properties are accurately known, and that, through a cut $\left(\tau_{N}^{\text {cut }}\right)$, allows one to translate an " $M$-parton" event to an " $N$-jet" event. The extra radiation is provided by a parton shower, which needs to be constrained by a requirement on $\tau_{N}^{\text {cut }}$.

- In UnNLOPS, one first promotes to NLO accuracy an "unitarized" CKKW approach, by carefully adding higher order contributions, and removing the pre-existing approximate terms at order $\alpha_{\mathrm{s}}$. The missing NNLO ingredients are then supplemented subsequently.

All the processes with 2 massless colored legs at $\mathrm{LO}$ can be described with $\mathrm{NNLO}_{\mathrm{QCD}}+\mathrm{PS}$ accuracy, and many results have been already obtained [2, 5-7, 11-28], including, in one case (based on the MiNLO' idea), results where NLO QCD accuracy was retained not only for the first jet, but also for the second one [29].

Besides the huge number of results for color-singlet production, in the last few months the first-ever $\mathrm{NNLO}_{\mathrm{QCD}}+\mathrm{PS}$ results for a process beyond color-singlet production were obtained: in Ref. [30] NNLO QCD corrections were matched to parton showers for top-pair production, through a non-trivial extension of the MINNLO $_{\mathrm{PS}}$ method. Recent progress with the Geneva method notably includes the first results obtained with a resolution parameter for the " 0 to 1 jet" region other than $\tau_{0}$ [25], where Radish results $[9,10,31]$ for $p_{t}$ resummation at N3LL were used as an input. In Ref. [32] the UnNLOPs method was generalized to take into account N3LO QCD corrections. Fig. 1 shows results for $t \bar{t}$ production (with MINNLOPS) and for the $p_{t}$ spectrum of the $Z$ boson in Drell-Yan production (with Geneva).

\footnotetext{
${ }^{1}$ After this talk was given, proof-of-concepts results using another $\mathrm{NNLO}_{\mathrm{QCD}}+\mathrm{PS}$ method were presented in Ref. [8].
} 

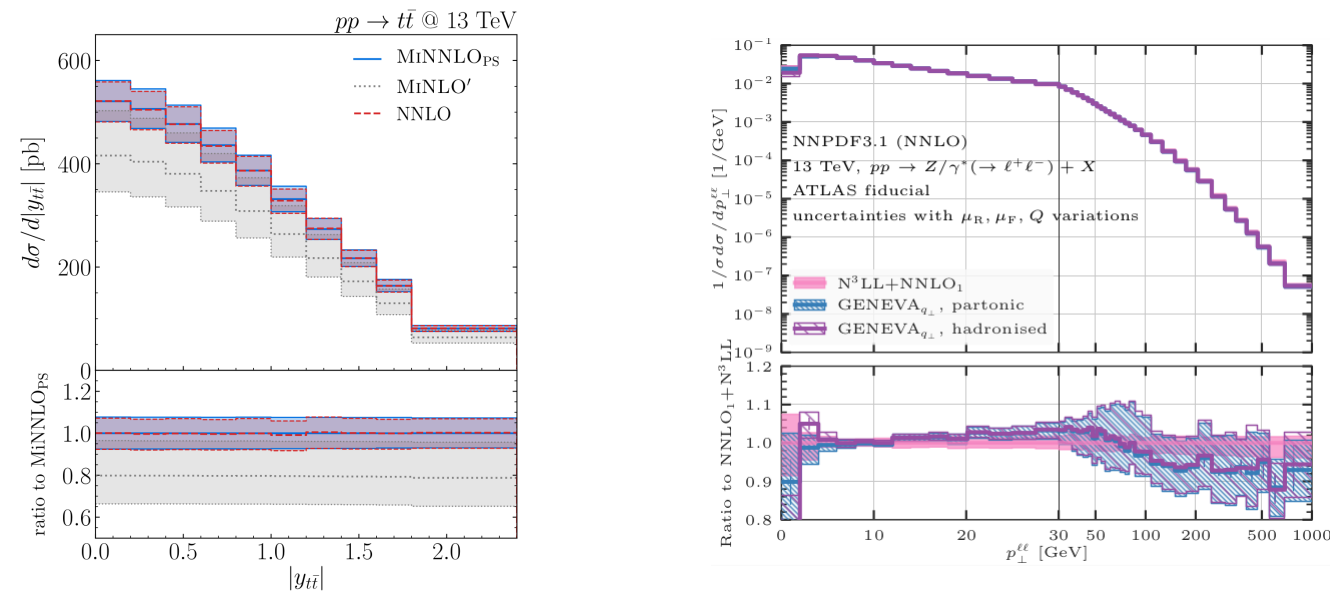

Figure 1: Left (supplemental material of Ref. [30]): comparison of NNLO (red), MiNLO' (gray), and

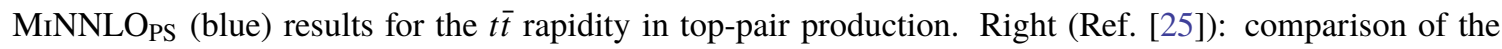
$p_{t}$ spectrum of the $Z$ boson in Drell-Yan production as obtained with Geneva (blue and violet) and with Radish+NNLOJET (pink).

\section{Matching NLO QCD and NLO EW corrections with Parton Showers}

The computation of NLO EW corrections to multileg processes at the LHC can be considered a conceptually solved problem. Building up from $\mathrm{NLO}_{\mathrm{QCD}}+\mathrm{PS}$ methods, it is possible to match NLO EW corrections with parton showers $\left(\mathrm{NLO}_{\mathrm{EW}}+\mathrm{PS}\right)$, using different approaches that also allow, at least for simple processes, a simultaneous matching of QCD and EW corrections. Nevertheless, it is certainly true that a fully-general approach to tackle this challenge, and that is valid also for processes featuring QCD/EW interference at $\mathrm{LO}$ (as, for instance, $p p \rightarrow t \bar{t}$ ) is still missing, and it is one of the open problems in the field.

There are currently two approaches to get $\mathrm{NLO}_{\mathrm{QCD}}+\mathrm{NLO}_{\mathrm{EW}}+\mathrm{PS}$ results:

- One can include EW corrections through a local $K$-factor (which relies on the use of approximated integrated real contribution, and that acts on the "Born" configurations only) and by adding real QED radiation only through the parton shower. The main limitation of this scheme, at times denoted as $\mathrm{EW}_{\mathrm{VI}}$ or EWvirt, and first proposed in Ref. [33], is that, formally, it is not valid for hard photon radiation. It has been successfully used, though, for several processes [33-37], and, notably, to incorporate approximate electroweak corrections in $\mathrm{NLO}_{\mathrm{QCD}}+\mathrm{PS}$ merged simulations [34, 35, 37].

- An exact matching of the EW corrections (both of virtual and real origin) can be obtained using the POWHEG-BOX-RES framework: this allows to include QCD and EW effects essentially through the traditional POWHEG approach, but also allowing for the generation of strong or electromagnetic real radiation from each resonance simultaneously. QCD and EW corrections are combined exactly (additively) at order $\alpha_{\mathrm{S}}$ and $\alpha_{\mathrm{EW}}$, whereas factorizable and mixed $\alpha_{\mathrm{S}}^{n} \alpha_{\mathrm{EW}}^{m}$ terms are only included in the collinear limit. Such approach has been used in Refs. [38-40] and, previously, for Drell-Yan production, in Refs. [41-46]. 
Two recent applications of the above methods are related to diboson production processes $(4 \ell$ production). Merged parton-shower predictions for $p \rightarrow W W$ and $p p \rightarrow W W+1$ jet production, that include NLO QCD and EW corrections (the latter in the EWvirt approximation), were presented by the authors of Ref. [37]. In Ref. [40], QCD and exact EW corrections to all 4-lepton final states were instead matched to parton showers through the refinements of the POWHEG method as implemented in the POWHEG-BOX-RES framework. Fig. 2 displays a couple of representative results taken from Ref. [40] (left) and [37] (right).
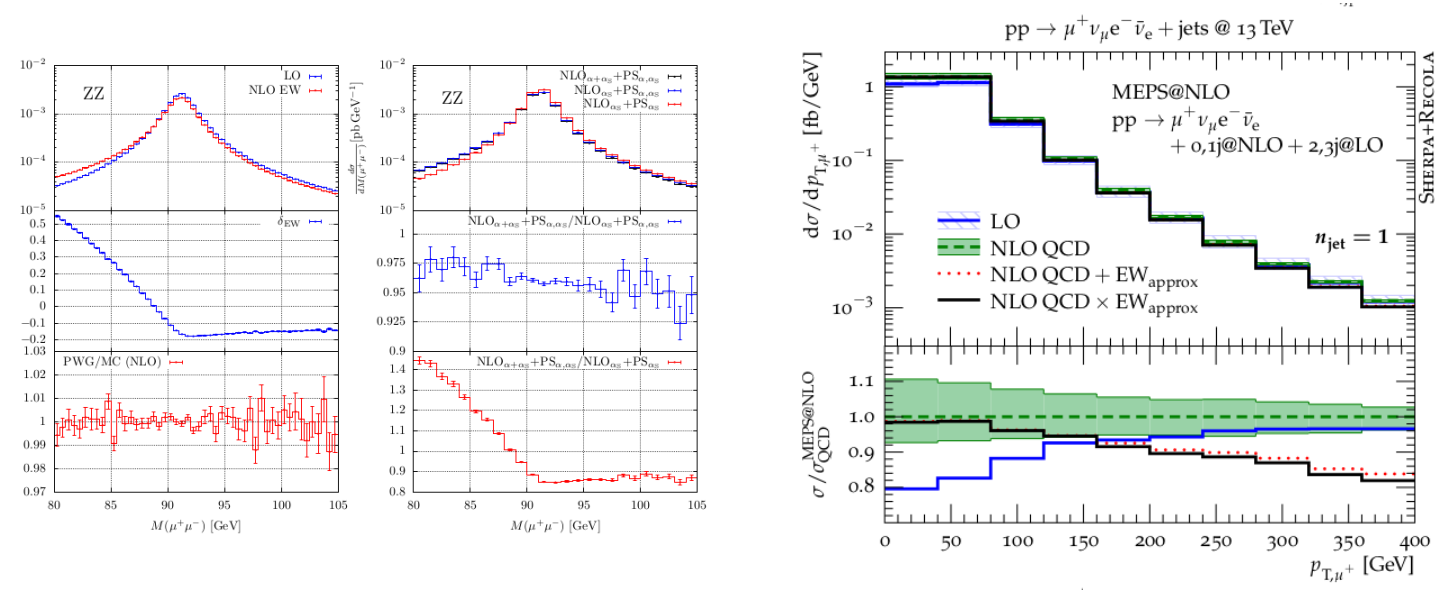

Figure 2: Left (Ref. [40]): Results, at different orders and approximations, for the invariant mass of the muonic pair in $p p \rightarrow e^{+} e^{-} \mu^{+} \mu^{-}$production. Right (Ref. [37]): Results with and without EW effects for the muon transverse momentum in NLO merged predictions for $p p \rightarrow \mu^{+} v_{\mu} e^{-} \bar{v}_{e}+$ jets.

Other schemes have been recently proposed to lift some of the limitations of the EWvirt approach: for instance, in Ref. [47], an EWsud scheme has been introduced, where LL and NLL EW corrections are included in the Sudakov limit [48], thereby allowing corrections to all jet multiplicities. In this context, even an hybrid scheme is being studied [49].

\section{Conclusions}

In this talk I summarized the recent activity in the context of matching parton showers with QCD fixed-order predictions at NNLO (NNLO $\mathrm{QCD}+\mathrm{PS})$, and of the matching of QCD and EW corrections simultaneously $\left(\mathrm{NLO}_{\mathrm{QCD}}+\mathrm{NLO}_{\mathrm{EW}}+\mathrm{PS}\right)$.

Among the future challenges, certainly there is the $\mathrm{NNLO}_{\mathrm{QCD}}+\mathrm{PS}$ matching for a process with jets at $\mathrm{LO}$, as well as the establishment of general method(s) to get $\mathrm{NLO}_{\mathrm{QCD}}+\mathrm{NLO}_{\mathrm{EW}}+\mathrm{PS}$ (and, eventually, $\mathrm{NNLO}_{\mathrm{QCD}}+\mathrm{NLO}_{\mathrm{EW}}+\mathrm{PS}$ ) accuracy for processes featuring $\mathrm{QCD} / \mathrm{EW}$ interference at $\mathrm{LO}$.

The focus of this review was on matching aspects. The improvement of parton-shower algorithms has been, recently, a very active area in the field. Such activity covers several directions, spanning from the inclusion of EW effects in parton showers to the introduction of a new generation of parton shower algorithms whose accuracy goes beyond the leading logarithm. It is likely that the impact of such developments (the last one in particular) will be significant in the future. I refer to the talks given, for instance, at the workshop "Taming the accuracy of event generators" [50], for a recent overview of this activity. 


\section{References}

[1] K. Hamilton, P. Nason, C. Oleari and G. Zanderighi, JHEP 1305, 082 (2013) [arXiv:1212.4504].

[2] K. Hamilton, P. Nason, E. Re and G. Zanderighi, JHEP 1310, 222 (2013) [arXiv:1309.0017 [hep-ph]].

[3] S. Alioli, C. W. Bauer, C. J. Berggren, A. Hornig, F. J. Tackmann, C. K. Vermilion, J. R. Walsh and S. Zuberi, JHEP 09 (2013), 120 [arXiv:1211.7049 [hep-ph]].

[4] S. Alioli, C. W. Bauer, C. Berggren, F. J. Tackmann, J. R. Walsh and S. Zuberi, JHEP 06 (2014), 089 [arXiv:1311.0286 [hep-ph]].

[5] S. Höche, Y. Li and S. Prestel, Phys. Rev. D 91 (2015) no.7, 074015 [arXiv:1405.3607 [hep-ph]].

[6] P. F. Monni, P. Nason, E. Re, M. Wiesemann and G. Zanderighi, JHEP 05 (2020), 143 [arXiv:1908.06987 [hep-ph]].

[7] P. F. Monni, E. Re and M. Wiesemann, Eur. Phys. J. C 80 (2020) no.11, 1075 [arXiv:2006.04133 [hep-ph]].

[8] J. M. Campbell, S. Höche, H. T. Li, C. T. Preuss and P. Skands, [arXiv:2108.07133 [hep-ph]].

[9] P. F. Monni, E. Re and P. Torrielli, Phys. Rev. Lett. 116 (2016) no.24, 242001 [arXiv:1604.02191 [hep-ph]].

[10] W. Bizon, P. F. Monni, E. Re, L. Rottoli and P. Torrielli, JHEP 02 (2018), 108 [arXiv:1705.09127 [hep-ph]].

[11] A. Karlberg, E. Re and G. Zanderighi, JHEP 09 (2014), 134 doi:10.1007/JHEP09(2014)134

[12] W. Astill, W. Bizon, E. Re and G. Zanderighi, JHEP 06 (2016), 154 [arXiv:1603.01620 [hep-ph]].

[13] W. Astill, W. Bizoń, E. Re and G. Zanderighi, JHEP 11 (2018), 157 [arXiv:1804.08141 [hep-ph]].

[14] E. Re, M. Wiesemann and G. Zanderighi, JHEP 12 (2018), 121 [arXiv:1805.09857 [hep-ph]].

[15] W. Bizoń, E. Re and G. Zanderighi, JHEP 06 (2020), 006 [arXiv:1912.09982 [hep-ph]].

[16] D. Lombardi, M. Wiesemann and G. Zanderighi, [arXiv:2010.10478 [hep-ph]].

[17] D. Lombardi, M. Wiesemann and G. Zanderighi, [arXiv:2103.12077 [hep-ph]].

[18] L. Buonocore, G. Koole, D. Lombardi, L. Rottoli, M. Wiesemann and G. Zanderighi, [arXiv:2108.05337 [hep-ph]]. 
[19] S. Höche, Y. Li and S. Prestel, Phys. Rev. D 90 (2014) no.5, 054011 [arXiv:1407.3773 [hep-ph]].

[20] S. Höche, S. Kuttimalai and Y. Li, Phys. Rev. D 98 (2018) no.11, 114013 [arXiv:1809.04192 [hep-ph]].

[21] S. Alioli, C. W. Bauer, C. Berggren, F. J. Tackmann and J. R. Walsh, Phys. Rev. D 92 (2015) no.9, 094020 [arXiv:1508.01475 [hep-ph]].

[22] S. Alioli, A. Broggio, S. Kallweit, M. A. Lim and L. Rottoli, Phys. Rev. D 100 (2019) no.9, 096016 [arXiv:1909.02026 [hep-ph]].

[23] S. Alioli, A. Broggio, A. Gavardi, S. Kallweit, M. A. Lim, R. Nagar, D. Napoletano and L. Rottoli, JHEP 04 (2021), 254 [arXiv:2009.13533 [hep-ph]].

[24] S. Alioli, A. Broggio, A. Gavardi, S. Kallweit, M. A. Lim, R. Nagar, D. Napoletano and L. Rottoli, JHEP 04 (2021), 041 [arXiv:2010.10498 [hep-ph]].

[25] S. Alioli, A. Broggio, A. Gavardi, S. Kallweit, M. A. Lim, R. Nagar, D. Napoletanog, C. W. Bauer and L. Rottoli, [arXiv:2102.08390 [hep-ph]].

[26] S. Alioli, A. Broggio, A. Gavardi, S. Kallweit, M. A. Lim, R. Nagar and D. Napoletano, Phys. Lett. B 818 (2021), 136380 [arXiv:2103.01214 [hep-ph]].

[27] T. Cridge, M. A. Lim and R. Nagar, [arXiv:2105.13214 [hep-ph]].

[28] Y. Hu, C. Sun, X. M. Shen and J. Gao, [arXiv:2101.08916 [hep-ph]].

[29] R. Frederix and K. Hamilton, JHEP 05 (2016), 042 [arXiv:1512.02663 [hep-ph]].

[30] J. Mazzitelli, P. F. Monni, P. Nason, E. Re, M. Wiesemann and G. Zanderighi, Phys. Rev. Lett. 127 (2021) no.6, 062001 [arXiv:2012.14267 [hep-ph]].

[31] W. Bizoń, X. Chen, A. Gehrmann-De Ridder, T. Gehrmann, N. Glover, A. Huss, P. F. Monni, E. Re, L. Rottoli and P. Torrielli, JHEP 12 (2018), 132 [arXiv:1805.05916 [hep-ph]].

[32] S. Prestel, [arXiv:2106.03206 [hep-ph]].

[33] S. Kallweit, J. M. Lindert, P. Maierhofer, S. Pozzorini and M. Schönherr, JHEP 04 (2016), 021 [arXiv:1511.08692 [hep-ph]].

[34] C. Gütschow, J. M. Lindert and M. Schönherr, Eur. Phys. J. C 78 (2018) no.4, 317 [arXiv:1803.00950 [hep-ph]].

[35] M. L. Czakon, C. Gütschow, J. M. Lindert, A. Mitov, D. Pagani, A. S. Papanastasiou, M. Schönherr, I. Tsinikos and M. Zaro, [arXiv:1901.04442 [hep-ph]].

[36] S. Kallweit, J. M. Lindert, S. Pozzorini and M. Schönherr, JHEP 11 (2017), 120 [arXiv:1705.00598 [hep-ph]]. 
[37] S. Bräuer, A. Denner, M. Pellen, M. Schönherr and S. Schumann, JHEP 10 (2020), 159 [arXiv:2005.12128 [hep-ph]].

[38] F. Granata, J. M. Lindert, C. Oleari and S. Pozzorini, JHEP 09 (2017), 012 [arXiv:1706.03522 [hep-ph]].

[39] M. Chiesa, A. Denner, J. N. Lang and M. Pellen, Eur. Phys. J. C 79 (2019) no.9, 788 [arXiv:1906.01863 [hep-ph]].

[40] M. Chiesa, C. Oleari and E. Re, Eur. Phys. J. C 80 (2020) no.9, 849 [arXiv:2005.12146 [hep-ph]].

[41] L. Barze, G. Montagna, P. Nason, O. Nicrosini and F. Piccinini, JHEP 04 (2012), 037 [arXiv:1202.0465 [hep-ph]].

[42] C. Bernaciak and D. Wackeroth, Phys. Rev. D 85 (2012), 093003 [arXiv:1201.4804 [hep-ph]].

[43] L. Barze, G. Montagna, P. Nason, O. Nicrosini, F. Piccinini and A. Vicini, Eur. Phys. J. C 73 (2013) no.6, 2474 [arXiv:1302.4606 [hep-ph]].

[44] C. M. Carloni Calame, M. Chiesa, H. Martinez, G. Montagna, O. Nicrosini, F. Piccinini and A. Vicini, Phys. Rev. D 96 (2017) no.9, 093005 doi:10.1103/PhysRevD.96.093005 [arXiv:1612.02841 [hep-ph]].

[45] A. Mück and L. Oymanns, JHEP 05 (2017), 090 [arXiv:1612.04292 [hep-ph]].

[46] M. Chiesa, F. Piccinini and A. Vicini, Phys. Rev. D 100 (2019) no.7, 071302 [arXiv:1906.11569 [hep-ph]].

[47] E. Bothmann and D. Napoletano, Eur. Phys. J. C 80 (2020) no.11, 1024 [arXiv:2006.14635 [hep-ph]].

[48] A. Denner and S. Pozzorini, Eur. Phys. J. C 18 (2001), 461-480 [arXiv:hep-ph/0010201 [hep-ph]].

[49] E. Bothmann, talk at "Parton Showers and Resummation" 2021 (PSR 2021), https://indico.cern.ch/event/1018828

[50] "Taming the accuracy of event generators", https://indico.cern.ch/event/999271/ 